Einleitung ............... 11

Abkürzungen ............... 13

Haemodynamik . . . . . . . . . . . 13

Phonokardiographie .............. . 13

Anatomische Bezeichnungen . . . . . . . . 14

Allgemeiner Teil . . . . . . . . . . . . . . 15

Methodik ................ 17

Phonokardiographie . . . . . . . . . . 17

Phonokardiographische Verfahren . . . . . . . . 18

Methoden der intrakardialen Phonokardiographie . . . 18

Unsere Technik der Registrierung . . . . . . . . . . 21

Phonokardiographische Nomenklatur . . . . . . . 23

Haemodynamik . . . . . . . . . . . 24

Intrakardialer Herzschall . . . . . . . . . . . . 28

Linkskardiale Phonokardiographie . . . . . . . . . 28

Rechtskardiale Phonokardiographie. . . . . . . . . 34

Projektion des intrakardialen Schalls

auf die Brustwand . . . . . . . . . . . . 36

Technische Aspekte der intrakardialen

Phonokardiographie . . . . . . . . . 39

Spezieller Teil . . . . . . . . . . . . . . 43

Normalperson . . . . . . . . . . . . . 45

Mitralstenose (MS) . . . . . . . . . . . . . . . . . . . 49

Mitralinsuffizienz (MI) . . . . . . . . . . . . 55

Mitralstenose mit Mitralinsuffizienz (MS + MI) . . . . 59

Mitralstenose mit Aorteninsuffizienz (MS + AI) . . . . 64

Mitralstenose mit Tricuspidalinsuffizienz (MS + AI) . . 69

Mitralstenose mit Mitralinsuffizienz und Aorteninsuffi-

zienz $(\mathrm{MS}+\mathrm{MI}+\mathrm{AI}) \ldots \ldots 72$
Introduction ................11

Abbreviations ............... 13

Hemodynamics .............. 13

Phonocardiography. ............ 13

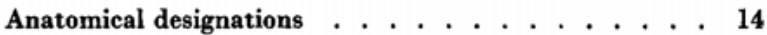

General part. . . . . . . . . . . . . 15

Methods ................ 17

Phonocardiography. . . . . . . . . . . . . 17

Phonocardiographic methods . . . . . . . . 18

Methods of intracardiac phonocardiography . . . . . 18

Our technique of registration . . . . . . . . . 21

Phonocardiographic nomenclature . . . . . . . . 23

Hemodynamics ............... 24

Intracardiac sounds and murmurs . . . . . . . . 28

Left heart phonocardiography . . . . . . . . . 28

Right heart phonocardiography . . . . . . . . . 34

Transmission of intracardiac sound

to the chest wall . . . . . . . . . . . . 36

Technical aspects of intracardiac phonocardiography . . 39

Special part . . . . . . . . . . . . . 43

Normal person . . . . . . . . . . . . . . 45

Mitral stenosis (MS) . . . . . . . . . . . . . 49

Mitral insufficiency (MI) . . . . . . . . . . . 55

Mitral stenosis with mitral insufficiency (MS + MI) . . 59

Mitral stenosis with aortic insufficiency (MS + AI) . . 64

Mitral stenosis with tricuspid insufficiency (MS + TI) . 69

Mitral stenosis with mitral insufficiency plus aortic insufficiency (MS + MI + AI) . . . . . . . . 72 
Mitralstenose und Aortenstenose mit Aorteninsuffizienz $(\mathrm{MS}+\mathrm{AS}+\mathrm{AI}) \ldots \ldots \ldots 75$

Aortenklappenstenose (AS) . . . . . . . . . . 81

Aorteninsuffizienz (AI) . . . . . . . . . . . . . 84

Aortenstenose mit Aorteninsuffizienz (AS + AI) . . . 87

Supravalvuläre Aortenstenose (Supravalv. AS) . . . . . 90

Subvalvulär-fibrotische Aortenstenose (FSS) . . . . 93

Idiopathische Herzhypertrophie, idiopathische hypertrophische Subaortenstenose, muskuläre Subaortenstenose (IH/IHSS, MSS) . . . . . . . . . . . . . . . 96

Aortenisthmusstenose (CoA) . . . . . . . . . . . 101

Hypertonie . . . . . . . . . . . . . . 105

Pericarditis constrictiva (Pc) . . . . . . . . . . 108

Pulmonalklappenstenose (PS) . . . . . . . . . . . . 111

Infundibuläre Pulmonalstenose (PS inf.) . . . . . . 114

Primäre pulmonale Hypertonie $(\mathrm{ppH}) \quad \ldots \quad$. . . . 117

Ductus arteriosus persistens (PDA) . . . . . . . . . 120

Vorhofseptumdefekt (ASD) . . . . . . . . . . . 123

Diagonaler Septumdefekt (DSD) . . . . . . . . . . . 126

Ventrikelseptumdefekt (VSD) . . . . . . . . . . . 129

Ventrikelseptumdefekt und infundibuläre Pulmonalstenose (VSD + PS inf.) . . . . . . . . . . . . 132

Ventrikelseptumdefekt mit schwerer pulmonaler Hypertonie (Eisenmenger - Syndrom) . . . . . . . . . . 136

Fallotsche Tetralogie (Fallot) . . . . . . . . . . . . 140

Mikro-Ventrikelseptumdefekt (VSD) . . . . . . . . . 143

Ventrikelseptumdefekt mit Pulmonalstenose und Aortenisthmusstenose (VSD + PS + CoA) . . . . . 147

Katheterrückzug . . . . . . . . . . . . . 150

Diastolischer Druckgradient . . . . . . . . . . . 151

Nierenarterienstenose . . . . . . . . . 152

Literatur . . . . . . . . . . . 153

Intrakardiale Phonokardiographie . . . . . . . . 153

Haemodynamik (Auswahl) . . . . . . . . . . . . 157

Monographien . . . . . . . . . . . 158
Mitral stenosis plus aortic stenosis with aortic insuffi-

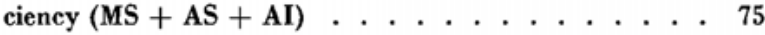

Valvular aortic stenosis (AS) . . . . . . . . . 81

Aortic insufficiency (AI) . . . . . . . . . . . . . . 84

Valvular aortic stenosis with aortic insufficiency (AS + AI) $\quad 87$

Supravalvular aortic stenosis (Supravalv. AS) . . . . . 90

Fibrous subaortic stenosis (FSS) . . . . . . . . . . . 93

Idiopathic hypertrophy, idiopathic hypertrophic subaortic stenosis, muscular subaortic stenosis (IH/IHSS, MSS) . . . . . . . . . . . . . . . . . . 96

Coarctation of the aorta $(\mathrm{CoA}) \ldots \ldots 101$

Hypertension . . . . . . . . . . . . . 105

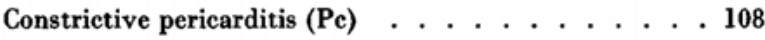

Valvular pulmonary stenosis (PS) . . . . . . . . . . 111

Infundibular pulmonary stenosis (PS inf.) . . . . . . . 114

Primary pulmonary hypertension $(\mathrm{ppH})$. . . . . . . 117

Patent ductus arteriosus (PDA) . . . . . . . . . . . 120

Atrial septal defect (ASD) . . . . . . . . . . . . . 123

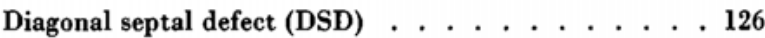

Ventricular septal defect (VSD) . . . . . . . . . . . . . 129

Ventricular septal defect and infundibular pulmonary stenosis (VSD + PS inf.) . . . . . . . . . . . . 132

Ventricular septal defect with pulmonary hypertension (Eisenmenger syndrome) . . . . . . . . . 136

Tetralogy of Fallot (Fallot) . . . . . . . . . . . . . 140

Ventricular septal micro-defect (VSD). . . . . . . . . 143

Ventricular septal defect with pulmonary stenosis and coarctation of the aorta (VSD + PS + CoA) . . . . 147

Pull-back of a catheter . . . . . . . . . . . . 150

Diastolic pressure gradient . . . . . . . . . . . 151

Renal artery stenosis . . . . . . . . . . . 152

Literature . . . . . . . . . . 153

Intracardiac Phonocardiography . . . . . . . . . . 153

Hemodynamics (selection) . . . . . . . . . . . 157

Monographs . . . . . . . . . . . . . . . . 158 
Die Vielzahl von Erkenntnissen pathophysiologischer und klinischer Art, die aus der Entwicklung moderner kardiodiagnostischer Methoden resultieren, erlauben eine erneute und präzisere Einschätzung der Wertigkeit althergebrachter klassischer Untersuchungsverfahren. Insbesondere wird durch die Einführung der intrakardialen Phonokardiographie die exakte Zuordnung der mit dem Hörrohr erhaltenen Befunde zu bestimmten physiologischen und pathophysiologischen Phänomenen möglich. Die intrakardiale Schallregistrierung im linken und rechten Herzen in Verbindung mit Druckmessungen, Dilutionskurven und angiokardiographischen Untersuchungen führt zu einer früher nicht geahnten Präzision der Diagnostik.

Verdienst der vorliegenden Monographie ist es, neben einer methodischen Einführung und einer umfassenden Literaturübersicht die Einarbeitung in diese komplizierte Materie anhand ausgewählter typischer Fälle von angeborenen und erworbenen Vitien zu ermöglichen und dabei dem Leser nicht nur beschreibend die Abweichungen der intra- und extrakardialen Ton- und Geräuschphänomene unter pathologischen Bedingungen nahe zu bringen, sondern ihn auch mit den Möglichkeiten der komplexen modernen Herzdiagnostik und ihrer pathophysiologischen Hintergründe vertraut zu machen.

Die umfassende Zusammenstellung von intrakardialen Schallkurven aus dem Herzen, die dank der ausgezeichneten Zusammenarbeit mit Prof. Porstmann gewonnen werden konnte, wird nicht nur für den Spezialisten, sondern auch für jeden kardiologisch interessierten Arzt wertvoll sein.
The extensive pathophysiological and clinical knowledge due to the development of modern cardiodiagnostic methods allows of a renewed and more precise evaluation of conventional examination techniques. By introducing intracardiac phonocardiography an exact attribution of findings obtained by means of the stethoscope to certain physiological and pathophysiological phenomena has been rendered possible. Intracardiac sound recording from both the left and right heart along with pressure measurements, dilution techniques and angiocardiographic examinations have resulted in a precision of diagnostics which was unthought of some years ago.

Besides a methodical introduction and a comprehensive bibliogaphy, the present monograph attempts to familiarise the reader with this complicated matter illustrated by typical cases of congenital and acquired heart diseases. However, the reader is not only acquainted, by way of description, with the alterations of intracardiac and extracardiac sound phenomena under pathological conditions, but also with the uses of complex modern cardiodiagnostics and its pathophysiological background. The extended compilation of intracardiac phonocardiograms from the heart obtained through the excellent co-operation with Professor Porstmann, is expected to be of some value to both the specialist and physician interested in cardiology.

H. DuTz

\section{H. DUTZ}


